Environment Conservation Journal 15 (1 \&2) 13-19, 2014

ISSN 0972-3099 (Print) 2278-5124 (Online)

Abstracted and Indexed

\title{
Application of CCME WQI to evaluate feasibility of potable water availability: A case study of Tehri dam reservoir
}

\author{
R. Bhutiani ${ }^{1} \bowtie$, D.R. Khanna ${ }^{1}$, Prashant Kumar Tyagi ${ }^{2}$ and Bharti Tyagi ${ }^{3}$
}

Received: 15.02.2014

Revised: 17.04.2014

Accepted: 01.05.2014

\begin{abstract}
In the present research work, the Canadian Council of Ministers of the Environment water quality index 1.0 (CCME WQI 1.0) was applied to assess water quality of Tehri dam reservoir by using the drinking water standard prescribed by the WHO (1999) and BIS (IS:10500, 1991). The physico-chemical parameters, ions concentration and heavy metals concentration used in the index calculation were total dissolved solids, $\mathrm{pH}$, alkalinity, dissolved oxygen, biochemical oxygen demand, chemical oxygen demand, total hardness, calcium, chloride, phosphate, sulphate, nitrate, total coliform (MPN/100 ml), turbidity, zinc, manganese, lead, nickel, iron and chromium. It was observed during the course of study that at all the four sites BOD, phosphate and total coliform showed greater deviation from the objective values. Total coliform was found to be more deviated from the normal values. Few important parameters were observed beyond the permissible limit for many times. The values of water quality index have shown that most of the sites are not fit for drinking purpose. Finally it was concluded that reservoir water should not be consumed for drinking purposes frequently without proper treatment.
\end{abstract}

Keywords: CCME WQI, BOD, TDS, pH, total coliform

\section{Introduction}

Water resource is critically important for living beings and developmental activities. Reservoirs, which are man-made lakes, are vital surface water resource that serve important environmental and economic purposes, including potable water supply, hydroelectric power generation, irrigation agriculture and fisheries. The desire and ability of building of dams to impound water by different civilizations dates back many millennia (Schnitter, 1994; McCully, 1996 and Uyigue, 2006). Reservoirs are normally more prone to get polluted as these are stagnant water bodies allowing more environmental exposure to stored water therein. Water quality deterioration in reservoirs usually comes from excessive nutrient inputs from agricultural fields, sewer discharge, inundated geological structures and organic decomposition of inundated biota. The chemical composition of reservoir water may depend on various factors such as topography, rainfall, geology, biology, temperature, land use, impact of humans etc. Poor

Author's Address

${ }^{1}$ Deptt. of Zoology and Env. Science' Gurukula Kangri University, Haridwar,

${ }^{2}$ Grass Roots Research and Creation India (P) Ltd. Noida,

${ }^{3}$ Uttaranchal College of Science and Tecnology, Dehradun Email: rbhutiani@gmail.com water quality can impair water use and affect other environmental interests, such as public health, fish and wildlife. So water quality has become increasingly more important in reservoir management for a number of reasons. All over the world, reservoirs are being harnessed for multiobjective demands, and thus, water demand attracts more attention causing water quality to draw frequent monitoring. Water quality for various uses is determined by the physical and chemical limnology of a reservoir and includes all physical, chemical and biological factors of water that influence the beneficial use of the water. The main problem in water quality monitoring is the complexity associated with analyzing the large number of data of measured variables containing rich information about the water resources (Saffran, 2001). The classification, modeling and interpretation of data are the most important steps in the assessment of water quality. Water quality index, excellent tool for classification, modeling and interpretation of data, provides a single number that expresses overall water quality in a given water basin, such as ponds, lake, river or stream at a time, based on several water quality parameters (Prasada et al., 2012). The WQI is basically a mathematical 
means of calculating a single value on certain scale from multiple test results that is understandable and used by the public.

\section{Material and Methods}

\section{Study Area}

The present research work is an attempt to assess utility potential of water quality for drinking purpose for many towns and villages located on the rim of reservoir. The Tehri Dam, main dam of Tehri hydro project complex, is $260.50 \mathrm{~m}$ ( 850 feet) high rock fill multipurpose river valley project dam constructed across the confluence of Bhagirathi and Bhilangana Rivers (major tributaries of River Ganga). The reservoir extends upto $45 \mathrm{kms}$ in the Bhagirathi valley and $25 \mathrm{kms}$ in the Bhilangana valley with a waterspread area of $42.5 \mathrm{sq} \mathrm{kms}$ in district Tehri Garhwal and District Uttarkashi of Bhagirathi Valley from Tehri town to Dharasu town. It impounds 3.22 million cubic meter of water covering an area of 5170.21 ha. The reservoir has a capacity to irrigate $2,70,000$ hectare of land. The project would have an underground power house with an installed capacity of 4 units of 250 MW each so that they can operate essentially to satisfy the peaking power requirements of Northern India. Four sampling sites, named Chinyali Sore, Chham, Koti, near the dam, have been taken in this research work on the reservoir as these sites are most accessible and suitable to reveal water quality of river.

\section{Sampling Procedure and analysis}

Water samples were collected once every month between 7.00 AM to 11.00 AM for one year from June 2009 to May 2010. The physico-chemical, ions concentration and heavy metals concentration were determined according to procedures outlined in Welch (1948), Trivedy and Goel (1990), APHA (1998) and Khanna and Bhutiani (2008). Total dissolved solids, $\mathrm{pH}$, Alkalinity, Dissolved oxygen, Biochemical oxygen demand, chemical oxygen demand, Total hardness, Calcium, Chloride, Phosphate, Sulphate, Nitrate, Total Coliform (MPN/100 ml) Turbidity (NTU), Zinc, Manganese, Lead, Nickel, Iron and Chromium were analysed during course of study.
Canadian Council of Ministers of the Environment (CCME) water quality index 1.0 In the present research work, the Canadian Council of Ministers of the Environment (CCME) water quality index 1.0 was applied by using the method described in Canadian water quality index 1.0 technical report and user's manual (2001) and CCME water quality index calculator 1.2. The index ranges from 0 to 100 . These numbers are divided into 5 descriptive categories to simplify presentation. Depending on the value, the water quality is characterized as excellent (95-100), good (80-94), fair (65-79), marginal (45-64) and poor (044). The details of the index are presented in Table 5. The CCME WQI model incorporates three measures of variance from selected water quality objectives (Scope; Frequency; Amplitude). These three factors combine to produce a value on a scale from 0 to 100 that represents the overall water quality. The formulation of the WQI as described in the Canadian Water Quality Index 1.0 - Technical Report (2001) is as follows. The measure for three factors are calculated as follows:

Factor 1: Scope F1 (Scope) represents the extent of water quality guideline non-compliance over the time period of interest.

$$
\mathrm{F}_{1}=\left(\frac{\text { Number of failed variables }}{\text { total number of variables }}\right) \times 100
$$

Factor 2: Frequency: $F_{2}$ (Frequency) represents the percentage of individual tests that do not meet objectives ("failed tests").

$$
\mathrm{F}_{2}=\left(\frac{\text { Number of failed tests }}{\text { total number of tests }}\right) \times 100
$$

Factor 3: Amplitude: $F_{3}$ (Amplitude) represents the amount by which failed test values do not meet their objectives. $F_{3}$ is calculated in three steps.

(I) The number of times by which an individual concentration is greater than (or less than, when the objective is a minimum) the objective is termed an "excursion" and is expressed as follows. When the test value must not exceed the objective:

$$
\text { excursion }_{i}=\left(\frac{\text { failed test }_{\text {value }}}{\text { objective }_{i}}\right)-1
$$


For the cases in which the test value must not fall below the objective:

$$
\text { excursion }_{i}=\left(\frac{\text { objective }_{i}}{\text { failed test value }_{i}}\right)-1
$$

(II) The collective amount by which individual tests are out of compliance is calculated by summing the excursions of individual tests from their objectives and dividing by the total number of tests (both those meeting objectives and those not meeting objectives). This variable, referred to as the normalized sum of excursions or $n s e$, is calculated as:

$$
\text { nse }=\frac{\sum_{i=1}^{n} \text { excursion }_{i}}{\text { numberoftests }}
$$

(III) $\mathrm{F}_{3}$ is then calculated by an asymptotic function that scales the normalized sum of the excursions from objectives (nse) to yield a range between 0 and 100.

$$
\mathrm{F}_{3}=\left(\frac{n s e}{0.01 n s e+0.01}\right)
$$

The CCME WQI is then calculated as:

$$
\text { CCME WQI }=100-\left(\frac{\sqrt{F_{1}^{2}+F_{2}^{2}+F_{3}^{2}}}{1.732}\right)
$$

The factor of 1.732 arises because each of the three individual index factors can range as high as 100 . This means that the vector length can reach as a maximum. Division by 1.732 brings the vector length down to 100 as a maximum. The divisor 1.732 normalises the resultant values to a range between 0 and 100, where 0 represents the "worst" water quality and 100 represents the "best" water quality.

$$
\sqrt{100^{2}+100^{2}+100^{2}}=\sqrt{30000}=173.20
$$

\section{Results and Discussion}

In this research paper the application of water quality index approach to Tehri dam reservoir had the purpose of providing a simple, valid method for expressing the results of several parameters in order to assess the water quality. Assembling different parameters into one single number leads an easy interpretation of index, thus providing an important tool for management purposes (Bordalo et al., 2001). The values of physico-chemical parameters, ions concentration and heavy metals concentration for all the four sampling site have been given in the tables 1 and 2. The $F_{1}, F_{2}$ and $F_{3}$ values and CCME WQI values are presented in the table 3. CCME WQI value ranges and categorization of water quality have been given in table 4 . Water quality standards for drinking purpose recommended by WHO (1999) and BIS (IS:10500, 1991) have been given in table 5 . The test values with grey background in table number 1 and 2 represent the failed values of the water quality variables. In CCME WQI model, the WQI value for first, second, third and fourth sampling sites was calculated to be 67, 64, 64 and 67 (table 3) respectively at Tehri dam reservoir for the study period (2009-2010). At first sampling site BOD, total coliform, lead and iron showed greater deviation from the objective values. At second sampling site BOD, total coliform and lead showed greater deviation from the objective values. At third sampling site BOD, phosphate, total coliform and iron showed greater deviation from the objective values. At fourth sampling site BOD, phosphate, total coliform and lead showed greater deviation from the objective values. Total coliform and BOD were found to be more deviated from the normal values at all sampling sites. TDS and turbidity showed deviation from objective values only in monsoon period. Remaining parameters did not show any deviation from objective values during whole study. Results, obtained from application of CCME by using the drinking water standard prescribed by the WHO (1999) and BIS (IS:10500, 1991), have shown values of WQI of sampling sites II and III belong to marginal class and are not fit for drinking purpose. While WQI of Sampling site I and IV fall in fair class but very close to marginal class. The above mentioned parameters, which deviated from the normal value mostly time, are mainly due to long time retention of Bhagirathi River's water before dam which receive heavy influx of various organic and inorganic substances from inundated trees, herbs, shrubs and geological structure in reservoir flood area. Besides all this, solid wastes and sewage are also produced from many residential colonies and villages situated on the river bank and reservoir rim. 
Bhutiani et al.

Table 1: Values of physico-chemical parameters, ions concentration and heavy metals concentration observed at sampling site I (Chinyali Sore) and II (Chham)

\begin{tabular}{|c|c|c|c|c|c|c|c|c|c|c|c|}
\hline 些 & Parameter & 莺它 & 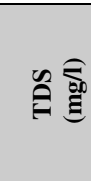 & 플 & ○ & 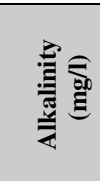 & ○ิ & 氶总 & 总 & 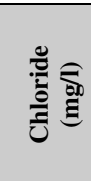 & 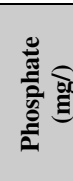 \\
\hline \multirow{12}{*}{ 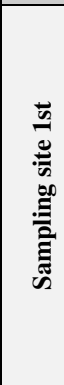 } & June & 4.14 & 612.00 & 7.60 & 8.91 & 55.00 & 2.43 & 106.00 & 23.25 & 15.76 & 0.285 \\
\hline & July & 6.24 & 448.00 & 7.40 & 9.18 & 65.00 & 3.10 & 98.66 & 21.64 & 22.43 & 0.043 \\
\hline & Aug. & 7.56 & 599.00 & 7.60 & 8.37 & 75.00 & 2.70 & 116.00 & 28.86 & 26.98 & 0.066 \\
\hline & Sep. & 6.25 & 154.00 & 7.80 & 9.18 & 65.00 & 2.70 & 82.00 & 20.84 & 22.43 & 1.679 \\
\hline & Oct. & 4.68 & 296.00 & 8.10 & 9.86 & 55.00 & 3.97 & 98.00 & 24.85 & 19.59 & 0.088 \\
\hline & Nov. & 4.78 & 148.00 & 7.90 & 10.13 & 47.50 & 2.84 & 74.00 & 20.84 & 15.47 & 0.577 \\
\hline & Dec. & 4.36 & 94.00 & 7.50 & 11.35 & 40.50 & 2.44 & 72.00 & 24.85 & 14.48 & 2.155 \\
\hline & Jan. & 1.58 & 69.00 & 7.10 & 10.21 & 41.50 & 1.89 & 65.00 & 23.25 & 10.22 & 0.042 \\
\hline & Feb. & 3.98 & 57.00 & 6.90 & 13.08 & 31.00 & 1.95 & 85.33 & 16.83 & 9.23 & 0.433 \\
\hline & March & 3.47 & 49.00 & 7.10 & 10.59 & 12.00 & 2.30 & 73.33 & 15.23 & 15.76 & 2.026 \\
\hline & April & 2.56 & 69.00 & 7.20 & 8.51 & 60.00 & 2.43 & 92.66 & 22.44 & 21.44 & 0.128 \\
\hline & May & 4.58 & 105.00 & 7.10 & 8.78 & 75.00 & 2.70 & 115.33 & 27.25 & 22.57 & 0.028 \\
\hline \multirow{12}{*}{ 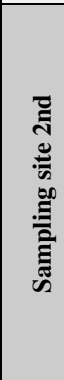 } & June & 2.50 & 84.00 & 7.50 & 7.91 & 45.50 & 2.43 & 110.66 & 20.84 & 22.29 & 0.155 \\
\hline & July & 3.26 & 58.00 & 7.80 & 8.72 & 47.00 & 3.83 & 107.33 & 22.44 & 29.67 & 0.177 \\
\hline & August & 5.80 & 169.00 & 8.10 & 9.18 & 55.00 & 3.10 & 112.00 & 24.85 & 28.54 & 1.485 \\
\hline & Sep. & 7.30 & 143.00 & 7.20 & 8.59 & 53.00 & 4.70 & 94.66 & 19.23 & 26.55 & 0.086 \\
\hline & Oct. & 3.00 & 122.00 & 7.40 & 9.27 & 43.00 & 3.98 & 106.00 & 23.24 & 16.47 & 1.273 \\
\hline & Nov. & 2.50 & 76.00 & 7.20 & 10.67 & 39.00 & 4.57 & 92.66 & 19.23 & 17.46 & 0.142 \\
\hline & Dec. & 3.56 & 38.00 & 7.10 & 9.48 & 28.50 & 1.76 & 74.00 & 20.04 & 19.45 & 2.631 \\
\hline & Jan. & 4.00 & 42.00 & 7.10 & 10.21 & 22.00 & 1.89 & 65.00 & 19.23 & 17.46 & 0.524 \\
\hline & Feb. & 4.50 & 58.00 & 7.10 & 10.35 & 27.50 & 2.84 & 62.00 & 16.83 & 12.49 & 0.436 \\
\hline & March & 3.60 & 31.00 & 7.10 & 10.13 & 37.50 & 2.84 & 86.66 & 19.23 & 16.47 & 0.839 \\
\hline & April & 2.40 & 44.00 & 7.00 & 7.24 & 31.50 & 2.97 & 103.33 & 19.23 & 22.29 & 0.128 \\
\hline & May & 2.40 & 52.00 & 7.20 & 7.83 & 49.00 & 2.97 & 103.33 & 23.24 & 22.57 & 1.125 \\
\hline
\end{tabular}

\begin{tabular}{|c|c|c|c|c|c|c|c|c|c|c|}
\hline 范 & Parameter & 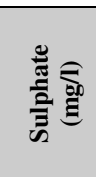 & 莺 & 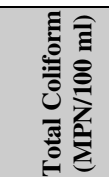 & : & 己气 & ప્ટ & 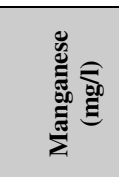 & 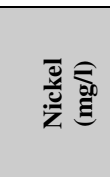 & 氞 \\
\hline \multirow{12}{*}{ 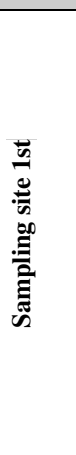 } & June & 9.65 & 0.115 & 220.00 & 0.1124 & 0.0391 & 0.4256 & 0.0087 & 0.0000 & 0.0000 \\
\hline & July & 8.54 & 0.097 & 359.00 & 0.0541 & 0.0124 & 0.4215 & 0.0058 & 0.0000 & 0.0000 \\
\hline & Aug. & 10.34 & 0.066 & 348.00 & 0.0912 & 0.0126 & 1.3698 & 0.0142 & 0.0000 & 0.0000 \\
\hline & Sep. & 12.46 & 0.258 & 410.00 & 0.0351 & 0.0071 & 0.5258 & 0.0135 & 0.0000 & 0.0000 \\
\hline & Oct. & 11.56 & 0.452 & 268.00 & 0.0345 & 0.0068 & 0.7568 & 0.0425 & 0.0000 & 0.0000 \\
\hline & Nov. & 12.46 & 0.234 & 378.00 & 0.0491 & 0.0042 & 0.1325 & 0.0358 & 0.0000 & 0.0000 \\
\hline & Dec. & 13.32 & 0.337 & 165.00 & 0.0421 & nil & 0.6658 & 0.0248 & 0.0047 & 0.0135 \\
\hline & Jan. & 17.18 & 0.326 & 314.00 & 0.0461 & 0.0156 & 0.3598 & 0.0159 & 0.0000 & 0.0000 \\
\hline & Feb. & 16.96 & 0.232 & 254.00 & 0.0000 & 0.0248 & 1.2484 & 0.0097 & 0.0000 & 0.0000 \\
\hline & March & 16.57 & 0.325 & 168.00 & 0.0624 & 0.0658 & 0.8369 & 0.0086 & 0.0000 & 0.0000 \\
\hline & April & 16.68 & 0.087 & 208.00 & 0.1124 & 0.0657 & 0.2369 & 0.0174 & 0.0000 & 0.0000 \\
\hline & May & 12.65 & 0.219 & 315.00 & 0.0561 & 0.0762 & 0.5427 & 0.0069 & 0.0238 & 0.0000 \\
\hline \multirow{12}{*}{ 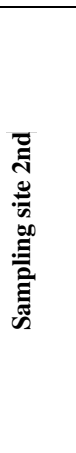 } & June & 8.62 & 0.126 & 350.00 & 0.1420 & 0.0482 & 0.3269 & 0.0958 & 0.0251 & 0.0000 \\
\hline & July & 12.25 & 0.155 & 421.00 & 0.0941 & 0.0503 & 0.6587 & 0.0894 & 0.0062 & 0.0000 \\
\hline & August & 12.15 & 0.247 & 265.00 & 0.0947 & 0.0415 & 0.2568 & 0.0561 & 0.0051 & 0.0000 \\
\hline & Sep. & 15.36 & 0.562 & 568.00 & 0.0752 & 0.013 & 1.1345 & 0.063 & 0.0082 & 0.0000 \\
\hline & Oct. & 20.13 & 0.465 & 647.00 & 0.0581 & 0.0145 & 0.1175 & 0.0932 & 0.0132 & 0.0000 \\
\hline & Nov. & 19.25 & 0.538 & 621.00 & 0.0924 & nil & 0.6256 & 0.0251 & 0.0091 & 0.0000 \\
\hline & Dec. & 22.45 & 0.222 & 715.00 & 0.0426 & 0.0151 & 0.2847 & 0.0045 & 0.094 & 0.0000 \\
\hline & Jan. & 16.36 & 0.325 & 625.00 & 0.0359 & 0.0142 & 1.1225 & 0.0098 & 0.0102 & 0.0059 \\
\hline & Feb. & 18.43 & 0.234 & 584.00 & 0.0654 & 0.0238 & 0.8446 & 0.0452 & 0.0062 & 0.0000 \\
\hline & March & 17.65 & 0.217 & 423.00 & 0.0814 & 0.0324 & 0.1248 & 0.0552 & 0.0084 & 0.0000 \\
\hline & April & 16.48 & 0.215 & 348.00 & 0.1320 & 0.0269 & 0.1358 & 0.044 & 0.0091 & 0.0000 \\
\hline & May & 17.45 & 0.318 & 498.00 & 0.1190 & 0.0425 & 0.2684 & 0.1561 & 0.0111 & 0.0000 \\
\hline
\end{tabular}

16

Environment Conservation Journal 
Application of CCME WQI to evaluate feasibility

Table 2: Values of physico-chemical parameters, ions concentration and heavy metals concentration observed at sampling site III (Koti) and IV (Near the dam)

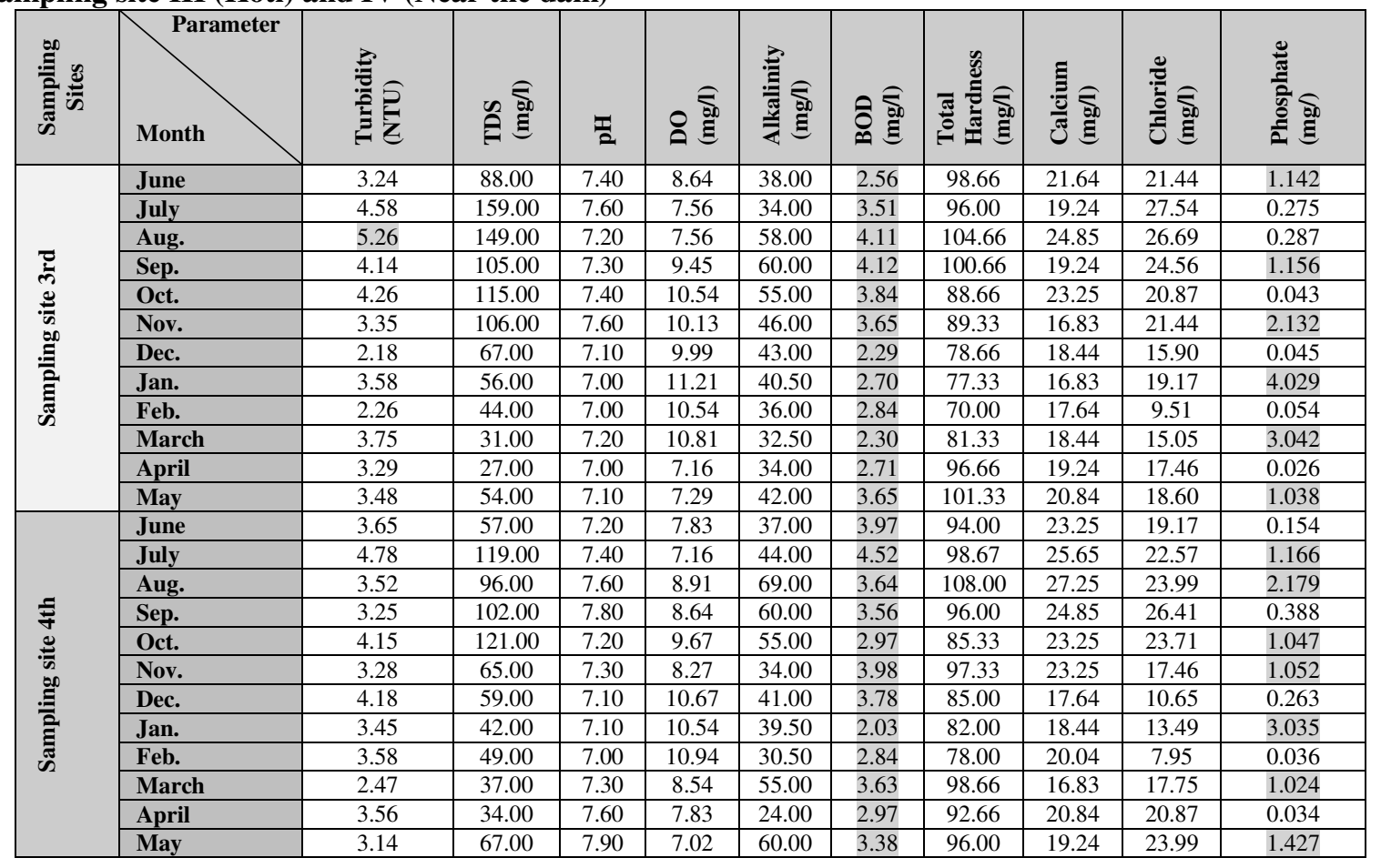

\begin{tabular}{|c|c|c|c|c|c|c|c|c|c|c|}
\hline 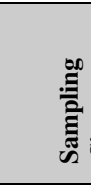 & Parameter & 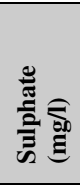 & 莺 & 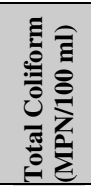 & 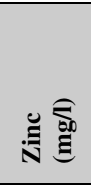 & 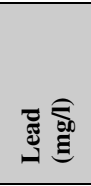 & 气 & 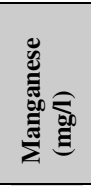 & 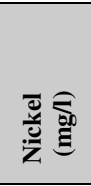 & 音 \\
\hline \multirow{12}{*}{ 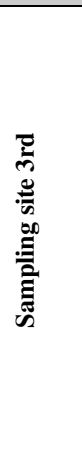 } & June & 10.35 & 0.095 & 425.00 & 0.0751 & 0.0000 & 1.2568 & 0.1430 & 0.0000 & 0.0000 \\
\hline & July & 13.14 & 0.142 & 485.00 & 0.0247 & 0.0000 & 0.4598 & 0.0957 & 0.0000 & 0.0000 \\
\hline & Aug. & 14.37 & 0.674 & 524.00 & 0.0269 & 0.0000 & 0.5458 & 0.0847 & 0.0000 & 0.0000 \\
\hline & Sep. & 14.56 & 0.402 & 345.00 & 0.0148 & 0.0000 & 0.8236 & 0.0714 & 0.0000 & 0.0000 \\
\hline & Oct. & 16.48 & 0.496 & 248.00 & 0.0329 & 0.0000 & 0.1475 & 0.0658 & 0.0000 & 0.0000 \\
\hline & Nov. & 24.15 & 0.359 & 541.00 & 0.0028 & 0.0000 & 0.7598 & 0.0428 & 0.0000 & 0.0000 \\
\hline & Dec. & 21.26 & 0.148 & 686.00 & 0.0035 & 0.0024 & 0.7359 & 0.0358 & 0.0016 & 0.0068 \\
\hline & Jan. & 19.47 & 0.097 & 574.00 & 0.0127 & 0.0000 & 1.2658 & 0.0047 & 0.0000 & 0.0000 \\
\hline & Feb. & 20.18 & 0.255 & 485.00 & 0.0369 & 0.0000 & 0.9475 & 0.0069 & 0.0000 & 0.0000 \\
\hline & March & 14.46 & 0.122 & 725.00 & 0.0478 & 0.0000 & 0.1369 & 0.0159 & 0.0000 & 0.0000 \\
\hline & April & 16.42 & 0.333 & 698.00 & 0.1290 & 0.0000 & 0.4256 & 0.0714 & 0.0000 & 0.0000 \\
\hline & May & 19.67 & 0.272 & 458.00 & 0.0098 & 0.0000 & 0.3697 & 0.0867 & 0.0000 & 0.0000 \\
\hline \multirow{12}{*}{ 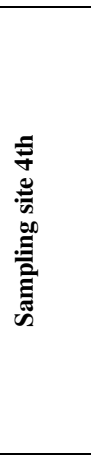 } & June & 10.95 & 0.132 & 269.00 & 0.0565 & 0.0658 & 0.5385 & 0.0358 & 0.0247 & 0.0000 \\
\hline & July & 12.45 & 0.284 & 541.00 & 0.0372 & 0.0458 & 0.1347 & 0.0475 & 0.0000 & 0.0000 \\
\hline & Aug. & 15.24 & 0.495 & 685.00 & 0.0254 & 0.0259 & 0.1268 & 0.0269 & 0.0000 & 0.0000 \\
\hline & Sep. & 19.06 & 0.582 & 425.00 & 0.0229 & 0.0026 & 1.1348 & 0.0095 & 0.0000 & 0.0000 \\
\hline & Oct. & 20.17 & 0.496 & 814.00 & 0.0387 & 0.0051 & 0.4715 & 0.0085 & 0.0000 & 0.0000 \\
\hline & Nov. & 19.48 & 0.545 & 759.00 & 0.0076 & 0.0035 & 1.4296 & 0.0126 & 0.0157 & 0.0000 \\
\hline & Dec. & 20.12 & 0.247 & 547.00 & 0.0283 & 0.0248 & 0.1187 & 0.0000 & 0.0000 & 0.0000 \\
\hline & Jan. & 18.43 & 0.348 & 469.00 & 0.0210 & 0.0369 & 0.7759 & 0.0041 & 0.0000 & 0.0044 \\
\hline & Feb. & 17.49 & 0.139 & 547.00 & 0.0081 & 0.0426 & 0.5642 & 0.0038 & 0.0000 & 0.0000 \\
\hline & March & 18.64 & 0.235 & 426.00 & 0.0226 & 0.0369 & 0.1383 & 0.0123 & 0.0000 & 0.0000 \\
\hline & April & 19.45 & 0.422 & 624.00 & 0.0310 & 0.0547 & 0.2411 & 0.0548 & 0.0258 & 0.0000 \\
\hline & May & 16.27 & 0.523 & 517.00 & 0.0351 & 0.0514 & 0.6262 & 0.0568 & 0.0000 & 0.0000 \\
\hline
\end{tabular}


Bhutiani et al.

Table 3: Value of CCME water quality index calculated for sampling sites

\begin{tabular}{|c|c|c|c|c|c|c|c|c|c|c|}
\hline & 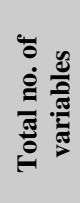 & 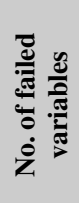 & 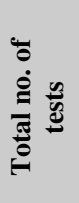 & 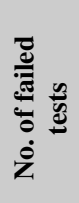 & 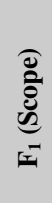 & 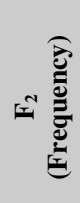 & 综 & 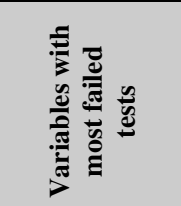 & $\bar{z}$ & 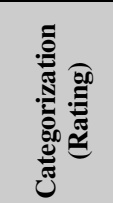 \\
\hline $\begin{array}{r}\text { Site } \\
\text { 1st }\end{array}$ & 19 & 8 & 228 & 48 & 42 & 22 & 31 & Total coliform & 67 & Fair \\
\hline $\begin{array}{l}\text { Site } \\
\text { 2nd }\end{array}$ & 19 & 8 & 228 & 48 & 42 & 21 & 41 & Total coliform & 64 & Marginal \\
\hline $\begin{array}{l}\text { Site } \\
\text { 3rd }\end{array}$ & 19 & 8 & 228 & 42 & 42 & 23 & 41 & $\begin{array}{c}\text { BOD } \\
\text { Total coliform }\end{array}$ & 64 & Marginal \\
\hline $\begin{array}{r}\text { Site } \\
\text { 4th }\end{array}$ & 19 & 6 & 228 & 49 & 32 & 22 & 44 & $\begin{array}{c}\text { BOD } \\
\text { Total coliform }\end{array}$ & 67 & Fair \\
\hline
\end{tabular}

Table-4: CCME WQI value ranges and categorization of water quality

\begin{tabular}{|c|l|c|l|}
\hline S.No. & Rating & \multicolumn{1}{|c|}{$\begin{array}{c}\text { CCME WQI } \\
\text { Value range }\end{array}$} & \multicolumn{1}{|c|}{ Categorization } \\
\hline $\mathbf{1}$ & Excellent & $95-100$ & $\begin{array}{l}\text { Water quality is protected with a virtual absence of threat or impairment; } \\
\text { conditions very close to natural or pristine levels. These index values can } \\
\text { only be obtained if all measurements are within objectives virtually all of } \\
\text { the time. }\end{array}$ \\
\hline $\mathbf{2}$ & Good & $80-94$ & $\begin{array}{l}\text { Water quality is protected with only a minor degree of threat or } \\
\text { impairment; conditions rarely depart from natural or desirable levels. }\end{array}$ \\
\hline $\mathbf{3}$ & Fair & $65-79$ & $\begin{array}{l}\text { Water quality is usually protected but occasionally threatened or } \\
\text { impaired; conditions sometimes depart from natural or desirable levels. }\end{array}$ \\
\hline $\mathbf{4}$ & Marginal & $45-64$ & $\begin{array}{l}\text { Water quality is frequently threatened or impaired; conditions often } \\
\text { depart from natural or desirable levels. }\end{array}$ \\
\hline $\mathbf{5}$ & Poor & $0-44$ & $\begin{array}{l}\text { Water quality is almost always threatened or impaired; conditions usually } \\
\text { depart from natural or desirable levels. }\end{array}$ \\
\hline
\end{tabular}

Table 5: Water quality standards for drinking purpose recommended by WHO (1999) and BIS (IS: 10500, 1991)

\begin{tabular}{|l|c|c|}
\hline Parameters & WHO & IS 10500 \\
\hline Taste & Agreeable & Agreeable \\
\hline TDS (mg/l) & 500.00 & 5.00 \\
\hline Turbidity (NTU) & - & 6.00 \\
\hline DO (mg/l) & - & 2.00 \\
\hline BOD (mg/l) & - & $6.50-8.50$ \\
\hline pH & $6.50-9.20$ & 200.00 \\
\hline Alkalinity (mg/l) & - & 250.00 \\
\hline Chloride (mg/l) & 250.00 & 200.00 \\
\hline Sulphate (mg/l) & 400.00 & 45.00 \\
\hline Nitrate (mg/l) & 45.00 & - \\
\hline Phosphate (mg/l) & - & 75.00 \\
\hline Ca ${ }^{++}$(mg/l) & 100.00 & 0.00 \\
\hline Total Coliform (MPN/100 ml) & 50.00 & 5.00 \\
\hline Zinc (mg/l) & 10.00 & 0.01 \\
\hline Lead (mg/l) & 0.01 & 0.30 \\
\hline Iron $(\mathbf{m g} / \mathbf{l})$ & 0.30 & 0.10 \\
\hline Manganese (mg/l) & 0.50 & 0.02 \\
\hline Nickel (mg/l) & 0.02 & 0.05 \\
\hline Chromium (mg/l) & 0.05 & \\
\hline
\end{tabular}


Kumar and Dua (2009) applied WQI method to Ravi river and found it one of most effective ways to communicate the information on water quality trends to the public or to the policy makers and water quality management. Devi et al. (2012) applied CCME Water Quality Index to the Lakes of Mandya, Karnataka State, India and found that water quality in the two lakes is degraded considerably due to anthropological activities. There are some limitations of WQI. For instance, WQI may not carry enough information about the real quality situation of the water. Also many uses of water quality data cannot be met with an index. But there are more advantages of WQI than disadvantages. An index is a useful tool for communicating water quality information to the public and to legislative decision makers. It is not a complex predictive model for technical and scientific application (McClelland, 1974).

\section{Conclusion}

Hence it is inferred from the results that overall quality of water of reservoir is not potable. Water quality is frequently threatened and impaired conditions often depart from natural and desirable level. It should not be used for drinking purposes frequently without proper treatment.

\section{References}

APHA, AWWA, WEF, 1998. Standards Methods for the Examination of Water and Wastewater, 20th edition, American Public, Health Association, American Water Works Association and Water Environmental Federation, Washington, DC 2005- 2605.

BIS. 1991. Indian standards: Drinking water specification, Bureau of indian standards, Manak Bhavan 9, Bahadur Shah Zafar Marg, New Delhi, 110002 India. IS: 10500:1991.

Bordalo, A.A., Nilsumranchit, W. and Chalermwat, K., 2001. Water quality and uses of the Bangpakong River (Eastern Thailand). Wat. Res. 35(15):3635-3642.
Canadian Council of Ministries of the Environment (CCMC), 2001. Canadian Water Quality Index 1.0 Technical Report and User's Manual. Canadian Environmental Quality Guidelines, Technical Subcommittee, Gatineau.

Devi Prasada, A.G., Kothathia, Siddaraju, 2012. Application of CCME water quality index (CWQI) to the Lakes of Mandya, Karnataka State, India. International Interdisciplinary Research Journal, II(I):108-114.

Khanna, D.R. and Bhutiani, R., 2008. Laboratory manual of water and wastewater analysis, Daya publishing house, Delhi, 1-189.

Kumar, Ashwani and Dua, Anish, 2009. Water quality index for assessment of water quality of River Ravi at Madhopur (India). Global Journal of Environmental Sciences, 8(1):49- 57.

McClelland, N.I., 1974. Water quality index application in the Kansas River basin prepared for the U.S. Environmental Protection Agency- Region 7. (EPA-907/9-74-001)

McCully P., 1996. Silenced Rivers: the Ecology and Politics of Large Dams, London, Zed Books.

Prasada, A., G., Devi and Kothathia, Siddaraju. 2012. Application of CCME water quality index (CWQI) to the Lakes of Mandya, Karnataka State, India. International Interdisciplinary Research Journal, 2(1):108-113, ISSN2249-9598.

Saffran K., 2001. Canadian water quality guidelines for the protection of aquatic life.

Schnitter, N., J., 1994. A History of Dams; The Useful Pyramids. Oregon, Books News Inc.

Trivedy, R.K. and Goel, P.K., 1990. Chemical and Biological methods for water pollution studies, Environmental publication, Karad, India.

Uyigue, Etiosa, 2006. Dams are unrenewable: A Discussion Paper. Community Research and Development Centre Nigeria.

Welch, P.S., 1948. Limnological methods. The Blakiston Co. Philadelphia, 1-381.

WHO, 1999. Guidelines for Drinking Water Quality. Second Edition. Geneva. 